\title{
Erythritol Chemical Structure, Biosynthesis Pathways, Properties, Applications, and Production
}

\author{
Osama Ibrahim \\ Biotechnology Consulting, Bio Innovation, Gurnee, United States
}

\section{Email address:}

Bioinnovation04@yahoo.com

\section{To cite this article:}

Osama Ibrahim Erythritol Chemical Structure, Biosynthesis Pathways, Properties, Applications, and Production. International Journal of Microbiology and Biotechnology. Vol. 6, No. 3, 2021, pp. 59-70. doi: 10.11648/j.ijmb.20210603.11

Received: June 21, 2021; Accepted: July 2, 2021; Published: July 9, 2021

\begin{abstract}
Erythritol is a natural four carbon sugar alcohol present in fruits, vegetables, mushrooms and in fermented foods. It is low calorie sweetener manufactured commercially by fermentation using osmophilic yeasts and yeasts-like fungi. Some filamentous fungi and heterofermentative lactic acid bacteria demonstrated on laboratory scale the production of erythritol at lower yield. Erythritol is gaining high market share as natural sweetener with applications in low calorie foods, beverages, and as an additive in combination with high intense zero calorie artificial sweeteners to enhance sweetness, texture, and to mask these artificial sweeteners bitter after taste. Due to increasing demand of erythritol with wide applications in foods, pharmaceuticals, and chemical industries, research activities are focusing on reducing production cost by fermentation conditions optimization. and improve industrial cultures via mutation or genetic engineering to utilize cheap and abundant byproducts such as crude glycerol and lignocellulosic materials as carbon sources in replacement to the costly glucose. Erythritol production with less intermediate metabolites in the fermentation process can be achieved by microbial metabolic pathway engineering. High yield of erythritol with less intermediate metabolites at the end of fermentation process will lead to improve erythritol recovery efficiency with higher yield and specifications.
\end{abstract}

Keywords: Sugar Alcohols, Osmiophilic Yeasts, Pentose Phosphate Pathway, Phosphoketolase Pathway, High Osmolarity Glycerol (HOG), Erythrose Reductase, Feed-batch Fermentation, Ion Exchange Chromatography

\section{Introduction}

Erythritol (1, 2, 3, 4-butanetetrol), is a four-carbon sugar alcohol (figure 1). It is a white crystal or powder anhydrous, and non-hygroscopic with similar appearance as table sugar sucrose. It has pleasant sweet taste with sweetness about $60-70 \%$ of sucrose and 0-0.2 calorie per gram comparing to 4 calorie per gram for sucrose [1]. It has solubility in water at room temperature at the rate of about 370 gram per liter. This water solubility rate increased by increasing the temperature. It has $\mathrm{PH}$ range from 2 to 10 depending on erythritol concentration in water. Its heat stability at a temperature over $160^{\circ} \mathrm{C}$, making erythritol suitable as low-calorie sweetener in baked goods [2]. Erythritol has synergistic sweetness effects with high intense artificial sweeteners such as sucralose, Stevioside (Stevia), aspartame, and acesulfame [3]. Erythritol is noncariogenic, has no glycemic response, not metabolize in digestive system, and almost non-laxative. Erythritol is mostly absorbed from small intestine to the blood stream and excreted in urine, small amount of non-absorbed erythritol might travel to the colon where it is resistant to fermentation by the colon microflora [4]. Erythritol is approved as generally recognized as safe (GRAS) in United States. Plus, approved as safe In European Union (E986), and in other countries [5].<smiles>OCC(O)C(O)CO</smiles>

Figure 1. Erythritol is a four-carbon sugar alcohol (polyol).

Erythritol has wide applications in low calories foods, baked goods, gums, confectionery, beverages, and as an excipient in pharmaceutical formulations such as medicated shewing gums, and coated tablets specially for moisture-sensitive medicines [6]. 
In addition, erythritol properties as anti-caries, and anti-oxidants make its applications in medicines and bio-chemical industry continue to expand. The average acceptable daily intake (ADI) of erythritol is 70 to 80 grams per day. Excessive intake of erythritol does not cause side effects and does not increase blood glucose due to its glycemic response is very low [6]. This low glycemic response properties making erythritol suitable for people suffering from diabetes, and obesity [7].

The common erythritol biosynthesis pathway in fermentation process from glucose or other carbon source using industrial cultures of yeasts or yeasts-like fungi is via the pentose-phosphate pathway. Erythritol biosynthesis pathway by bacteria such as heterofermentative lactic acid is via phosphoketolase pathway. These two pathways for erythritol biosynthesis are multiple steps of enzymatic reactions generate intermediate metabolites effects the rate of erythritol biosynthesis plus interfere in erythritol recovery efficiency from fermentation broth. To Improve erythritol yield and recovery efficiency microbial mutation or genetic engineering is nesseacary to reduce or block these intermediate metabolites biosynthesis.

\section{Technology Aspects}

Extraction of erythritol from natural sources such as fruits is not practical due to its low concentration in these natural sources. Chemical synthesis of erythritol was investigated and demonstrated to be very costly due to low yield, and the requirement of high temperature in the presence of catalyst such as the metal nickel [8]. The detection of low concentration of erythritol in blackstrap molasses fermented by osmophilic yeasts in the year 1950 open the possibility for the production of erythritol by microbial fermentation.

A wide variety of osmophilic yeasts and yeasts-like fungi from genus Moniliella, Torula, Candida, Pichia, Trichoderma, and Yarrowia are found to produce erythritol from glucose. Currently erythritol is manufactured on large scale from glucose as carbon source using osmophilic yeasts and yeasts-like fungi strains of,
Moniliella pollinis, Trichosporonoides megachiliensis, and Yarrowia lipolytic. Glucose as a carbon source is used at high concentration by these osmophilic yeasts and yeasts-like fungi strains in fermentation process for high production yield of erythritol [9]. Glucose is produced enzymatically from corn or other starch crops by acid or enzymatic hydrolysis [10]. Other carbon sources such as fructose, sucrose, and glycerol were also, investigated in fermentation process for erythritol production and can be used on large scale production. The disaccharide sucrose as a carbon source requires enzymatic hydrolysis by invertase enzyme into monosaccharides glucose and fructose for the conversion of these two monosaccharides into erythritol by fermentation process.

\subsection{Biosynthesis Pathways}

Erythritol biosynthesis pathway from glucose using osmophilic yeasts or yeasts-like fungi is via pentose phosphate pathway [11]. This pathway has two phases of oxidative phase, and non-oxidative phase (figure 2).

Oxidative phase: In this phase two molecules of $\mathrm{NADP}^{+}$are reduced to two NADPH as a source of energy for the conversion of glucose-6-P into ribulose-5-P in the following biosynthesis cycle (figure 3):

Glucose is phosphorylated into glucose-6-P by the enzyme glucose kinase. Glucose-6-P inter pentose-phosphate pathway and in the presence of $\mathrm{NADP}^{+}$, is converted into 6-glucose-delta-lactone by the enzyme glucose-6-phosphate dehydrogenase. The generated 6-glucose-delta-lactone in the presence of water is converted into 6-phosphogloconate and $\mathrm{H}^{+}$by the enzyme lactonase. The generated 6-phosphogloconate in the presence of $\mathrm{NADP}^{+}$is converted into ribulose-5-P, NADH, and $\mathrm{H}^{+}$by the enzyme phospho-gluconate dehydrogenase [12]. All these steps in oxidative phase of pentose-phosphate pathway are irreversible enzymatic reactions, and are essential for the next non-oxidative phase for erythritol biosynthesis.

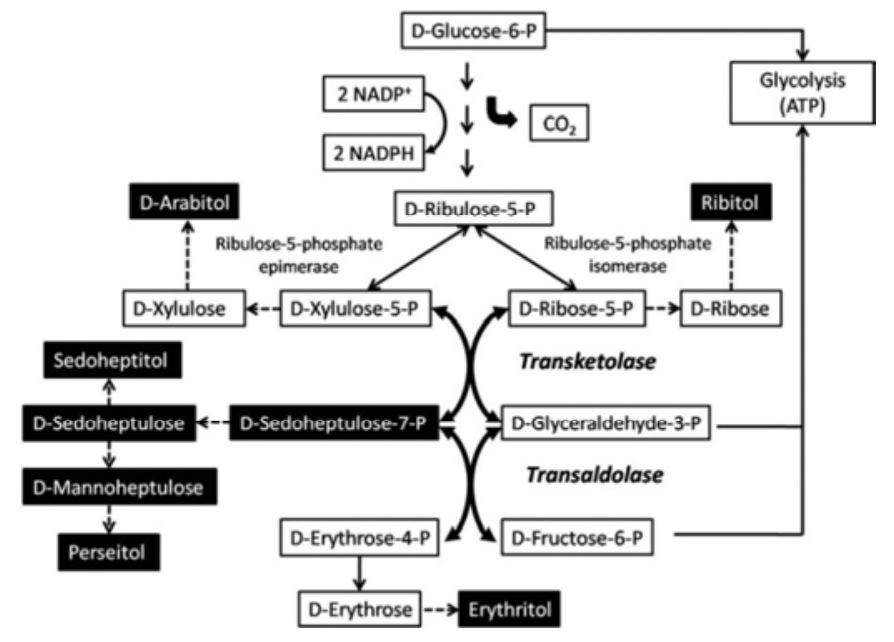

Figure 2. Pentose Phosphate pathway.

Pentose phosphate pathway is the main pathway for the conversion of phosphorylate glucose (glucose-6-P) into erythritol by fermentation process using osmophilic yeasts and yeasts- like fungi [49] 


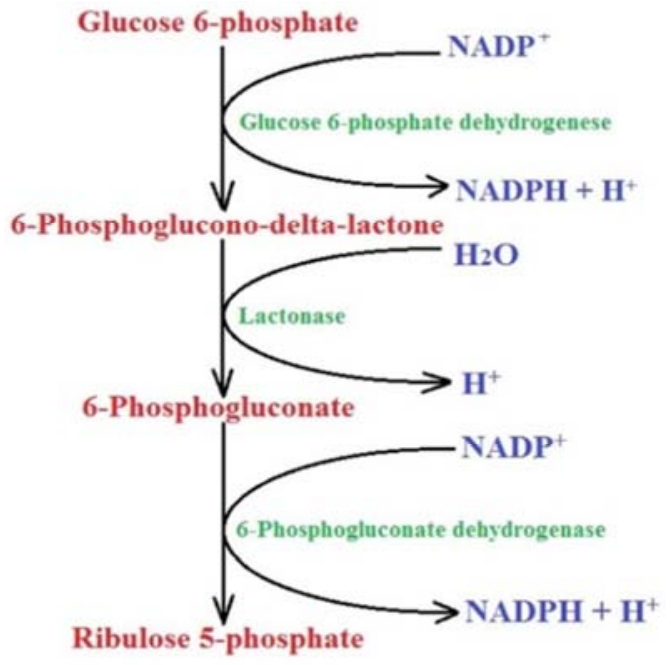

Figure 3. Pentose Phosphate Pathway (oxidative phase).

The conversion of glucose-6-phosphate into ribulose-5-phosphate by the two enzymes glucose-6-phosphate dehydrogenase, and 6-phoshpgluconate dehydrogenase in the presence of two molecules of co-enzyme NADP reduced to tow molecules NADPH and two $\mathrm{H}^{+}$[this image is copied from microscopic master.com]
Non-oxidative phase: This phase link glycolytic pathway to the pentose-phosphate pathway, and contains key enzymes of phosphopentose isomerase, phosphopentose epimerase, transketolase and transaldolase [13]. This non-oxidative phase is reversable enzymatic reactions and started from ribulose-5-P as a substrate for the conversion into erythrose-4-P in the following biosynthesis cycle (figure 4):

Ribulose-5-P is converted into ribose-5-P and xylulose-5-P by two-enzymes ribulose-5-phosphate epimerase, and ribulose-5-phosphate isomerase. Ribose-5-P plus xylulose-5-P reacted together enzymatically and are converted into the seven carbons of sedoheptulose-7-P and the three carbons of glyceraldehyde-3-P by the enzyme transketolase. Both generated Sedoheptulose-7-P plus glyceraldehyde-3-P are converted into erythrose-4-P and fructose-6-P by the enzyme transaldolase. The generated fructose-6-P is converted into erythrose-4-P and acetyl-2-P by the enzyme transketolase (figure 5). Both generated erythrose-4-P are converted into erythrose in the presence ADP by the enzyme phosphatase. Finally, generated erythrose in the presence of NADPH is converted into erythritol and $\mathrm{NADP}^{+}$by the enzyme erythrose reductase.

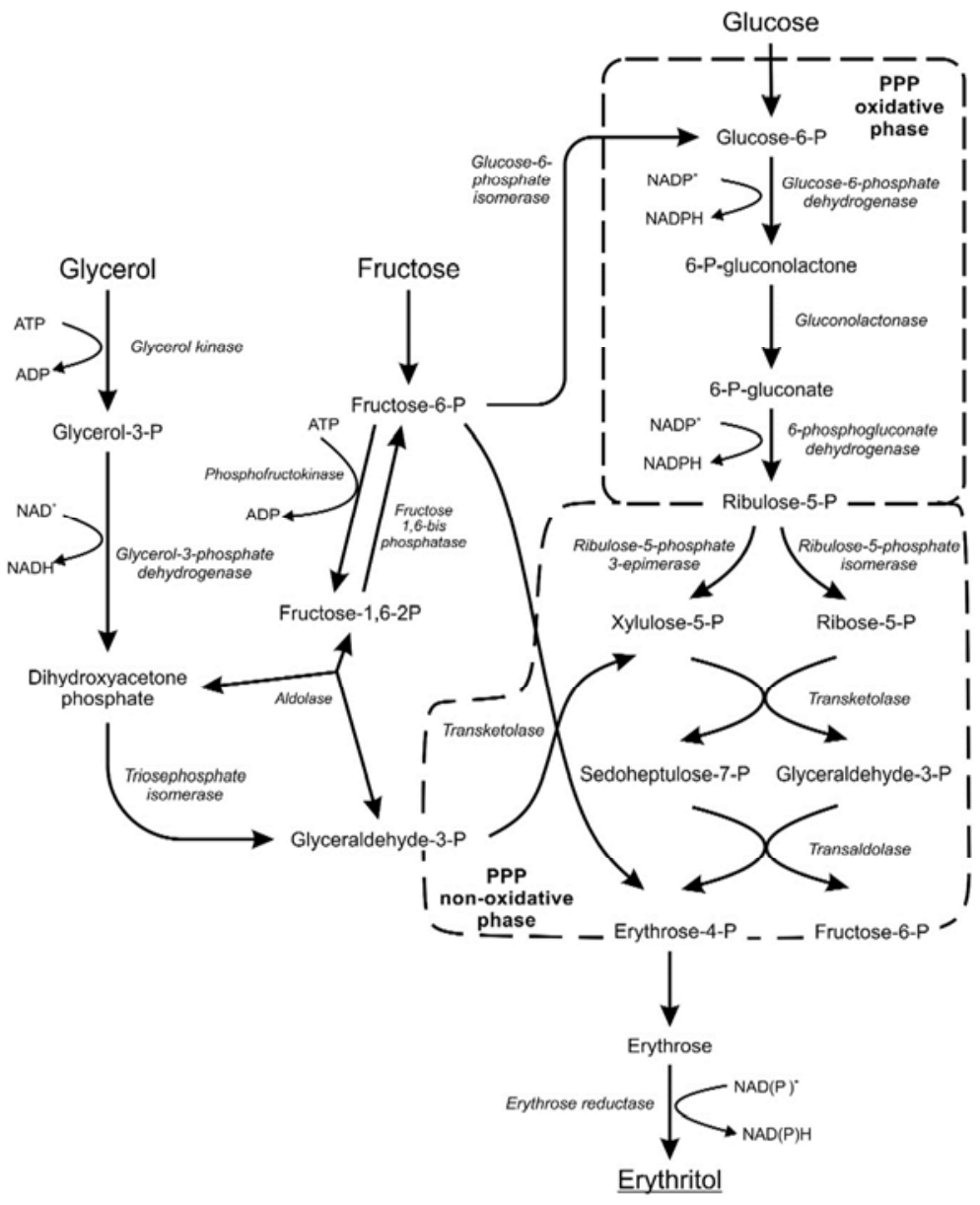

Figure 4. Pentose phosphate pathway for the conversion of glucose, fructose, and glycerol into erythritol.

Non-oxidative phases in pentose phosphate pathway link glycolytic pathway to pentose phosphate pathway to convert ribulose-5-P into erythritol using key enzymes of ribulose-5-phospate epimerase, ribose 5-phosphate isomerase, transketolase, transaldolase, and erythrose reductase [31]. 


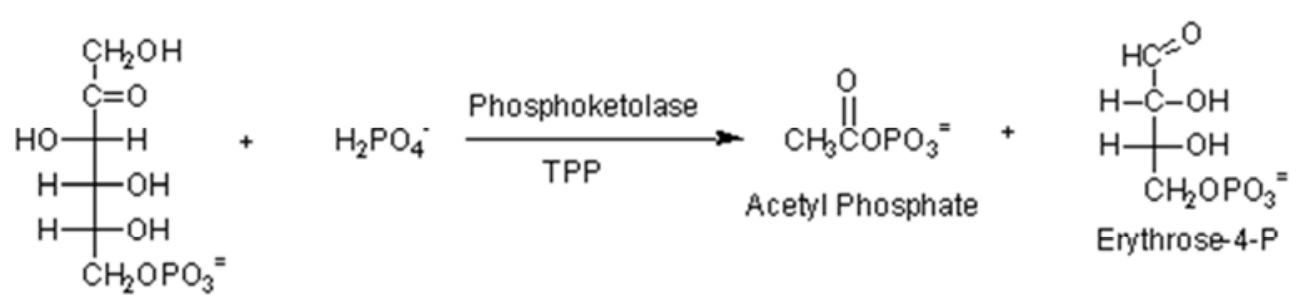

Fructose-6-P

Figure 5. Conversion of Fructose-6-Phosphate into Erythrrose-4-Phosphate.

Fructose-6-Phosphate is converted into erythrose -4-P and acetyl-3-P by the enzyme transketolase. Erythrose-4-P by the enzyme phosphatase in the presence of ADP is converted into erythrose plus ATP. Generated erythrose is converted into erythritol by the enzyme erythrose reductase in the presence of the co-enzyme NADPH.

In summary, high activity of key enzymes in pentose phosphate pathway are responsible for the production of high yield of erythritol from glucose. These key enzymes are glucose-6-phosphate dehydrogenase, phosphogluconate dehydrogenase, transketolase, transaldolase, and erythrose reductase. It is important to highlight that the enzyme erythrose reductase is not present in most yeasts or yeasts-like fungi, but it is present in Moniliella megachiliensis, Yarrowia lipolytica, Torula sp. and Candida magnoliae making these yeast cultures more desirable for erythritol production [14]. In addition, to the conversion of glucose into erythritol, these yeasts and yeasts-like fungi cultures, the yeast culture of Candida magnoliae demonstrated erythritol biosynthesis by fermentation process from fructose as a carbon source with higher yield than glucose [15].

Utilization of the disaccharide sucrose as a carbon source for erythritol production in fermentation process is limited to few yeast cultures that express the intracellular enzyme invertase to hydrolyze the glycosidic bond in the disaccharide sucrose into the two monosaccharides glucose and fructose, followed by the conversion of these two monosaccharides into erythritol via pentose-phosphate pathway [16].

The production of biodiesel from fat and oil by esterification process in the presence of methanol, resulted in the production of alkyl ester (biodiesel) and crude glycerol as a byproduct (figure 6). Utilization of this crude glycerol as a cheap carbon source for the production of erythritol by fermentation process has been investigated. Some selected osmophilic yeast mutants such as Yarrowia lipolytica demonstrated the capability to produce erythritol from glycerol by fermentation at $\mathrm{PH}$ 3.0. This low $\mathrm{PH}$ in fermentation process is an advantage for protecting fermentation process from microbial contamination [17].

Pentose-phosphate pathway for the conversion of glycerol into erythritol (figure 7) is started by the phosphorylation of glycerol into glycerol-3-P by the enzyme glycerol kinase [18]. The generated glycerol-3-P inter glycolysis pathway and is converted into glyceraldehyde-3-P by the enzyme glycerol-3-phosphate dehydrogenase or by triosephosphate isomerase (both enzymes are present in glycolysis and in gluconeogenesis pathways). In this step, some of glyceraldehyde-3-P is converted into fructose-1,6-bisphosphate, then into fructose-6-P. Both generated gyceraldehyde-3-P and fructose-6-P inter the non-oxidative phase in pentose-phosphate pathway as substrates for the formation of erythrose-4-P. Finally, erythrose-4-P is converted into erythritol as previously shown in pentose-phosphate pathway from glucose. It is important to highlight that the oxidative phase in pentose-phosphate pathway is not involve in this glycerol pathway into erythritol, even though it has been demonstrated that overexpression of oxidative pathway enzymes glucose-6-phosphate dehydrogenase and 6-phos-phogluconate dehydrogenase in the yeast Yarrowia lipolytica improved the production of erythritol from glycerol [19].<smiles>[R]C(=O)OCC(COC([R])=O)OC([R])=O</smiles>

Triglyceride

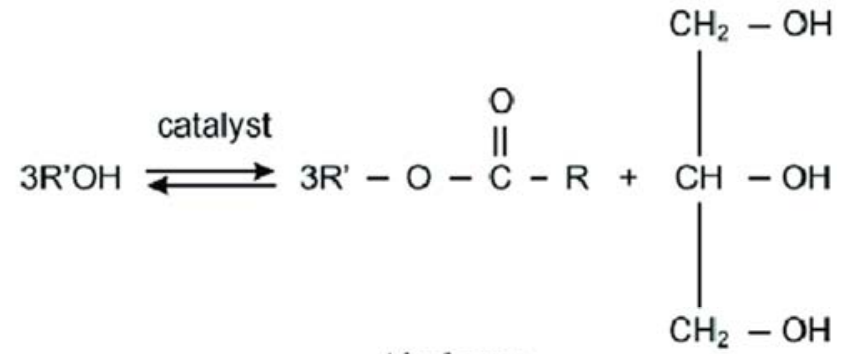

Alcyl ester (Biodiesel)
Glycerol

Figure 6. Transesterification reaction of biodiesel production.

The chemistry for biodiesel production from triglycerides is by using methanol and the catalyst nickel to produce Alkyl ester (biodiesel), and crude glycerol. This crude glycerol as byproduct can be used for the production of erythritol by fermentation process [50] 


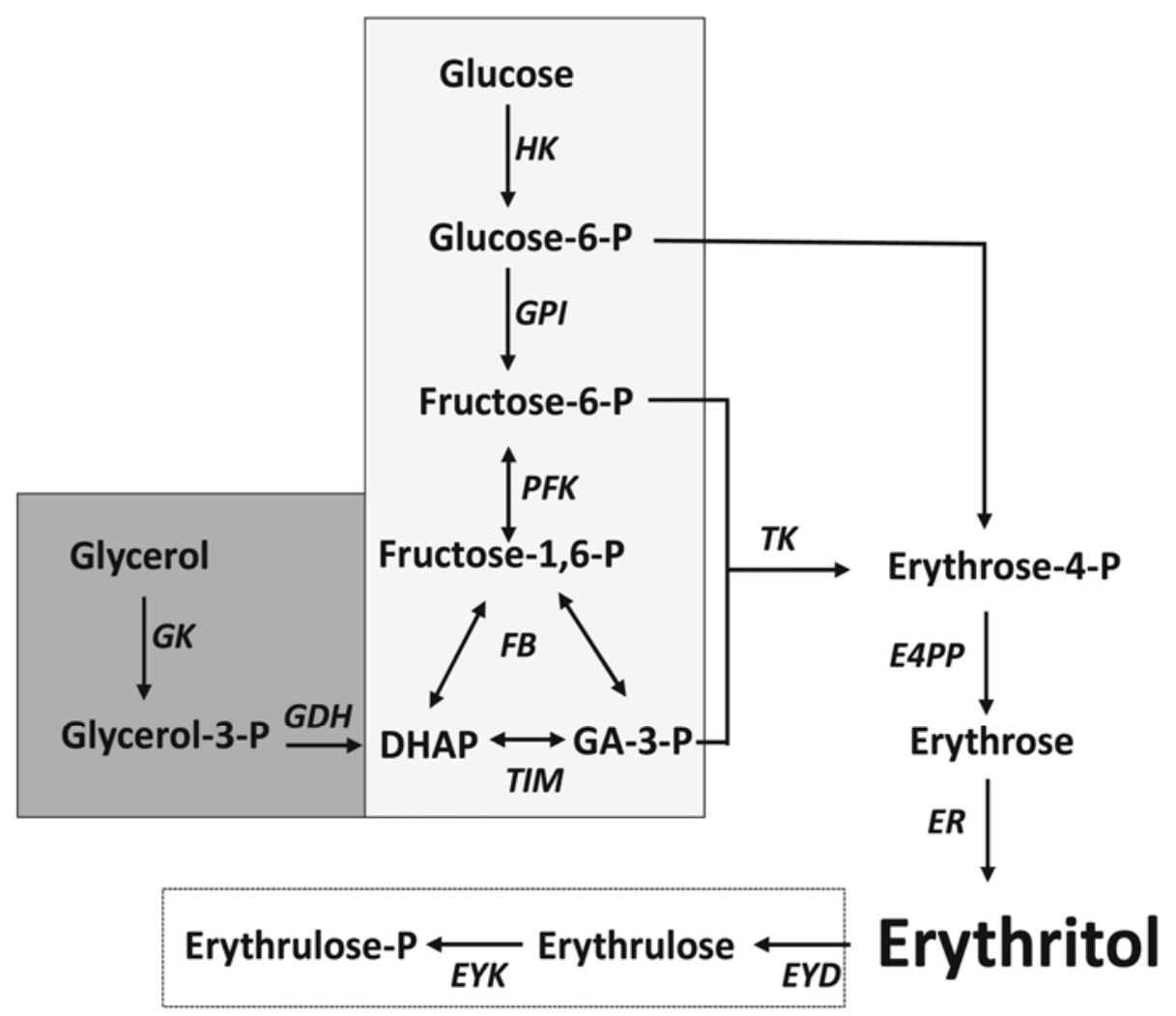

Figure 7. Glycerol pathway to erythritol.

Glycerol is phosphorylation into glyceraldeyde-3-P by the enzyme glycerol kinase, glyceraldehyde -3-P is dehydrogenated into dihydroxyacetone phosphate (DHAP) by the enzyme glycerol-3-Phosphate dehydrogenase. The biosynthesis of erythritol is from dihydroxyacetone Phosphate (DHAP) via non-oxidative phase in pentose phosphate pathway [51].

\subsection{Non-yeast Producing Erythritol}

In addition, to commercial production of erythritol on large scale by fermentation process using osmophilic yeasts and yeasts-like fungi, other non-yeast microorganisms of filamentous fungi, and heterofermentative lactic acid bacteria demonstrated in laboratory scale to produce low yield of erythritol. The following are examples:

\section{Trichoderma reesei}

This filamentous fungus is capable to produce erythritol via pentose-phosphate pathway, plus express the key enzyme erythrose reductase as intracellular enzyme for the reduction of generated erythrose into erythritol. These filamentous fungi, also express cellulase and hemicellulose enzymes as extracellular enzymes, to hydrolyze lignocellulosic materials into monosaccharides of hexoses and pentoses. The capability of filamentous fungi such as Trichoderma reesei strain to hydrolyze cellulose and hemicellulose in lignocellulosic materials into monosaccharides give these filamentous fungi an advantage in utilizing agriculture crops byproducts, and industrial biowastes rich in cellulose and hemicellulose as a cheap carbon source for erythritol production [20]. Erythritol pathway by these filamentous fungi is also, via the pentose phosphate pathway, similar to osmophilic yeasts and yeasts-like fungi producing erythritol on large scale previously describes. Erythritol yield from these filamentous fungi is very low comparing to industrial osmophilic yeasts, and yeast-like fungi [21]. Future potential erythritol production on large scale from cheap lignocellulosic materials as carbon sources can be achieved by microbial mutation or genetic engineering for overexpression pentose-phosphate pathway key enzymes in these filamentous fungi cells for high conversion rate of lignocellulosic materials into erythritol.

Leuconostoc oenos

The production of erythritol from glucose or fructose by heterofermentative lactic acid bacteria such as Leuconostoc oenos was investigated in laboratory scale [22]. The biosynthesis of erythritol by Leuconostoc oenos is through phosphoketolase pathway (figure 8). This pathway under anaerobic microbial fermentation conditions, started by the phosphorylation of glucose into glucose-6-P by the enzyme glucose kinase in glycolysis pathway. The generated glucose-6-P inter phoshopketolase pathway and isomerized into fructose-6-P. The generated fructose-6-P is cleaved into erythrose-4-P and acetyl-2-P, by the enzyme transketolase. The generated erythrose-4-P is converted into erythrose by phosphatase enzyme, followed by the conversion of erythrose into erythritol by the enzyme erythrose reductase in the presence of NADPH [23]. 


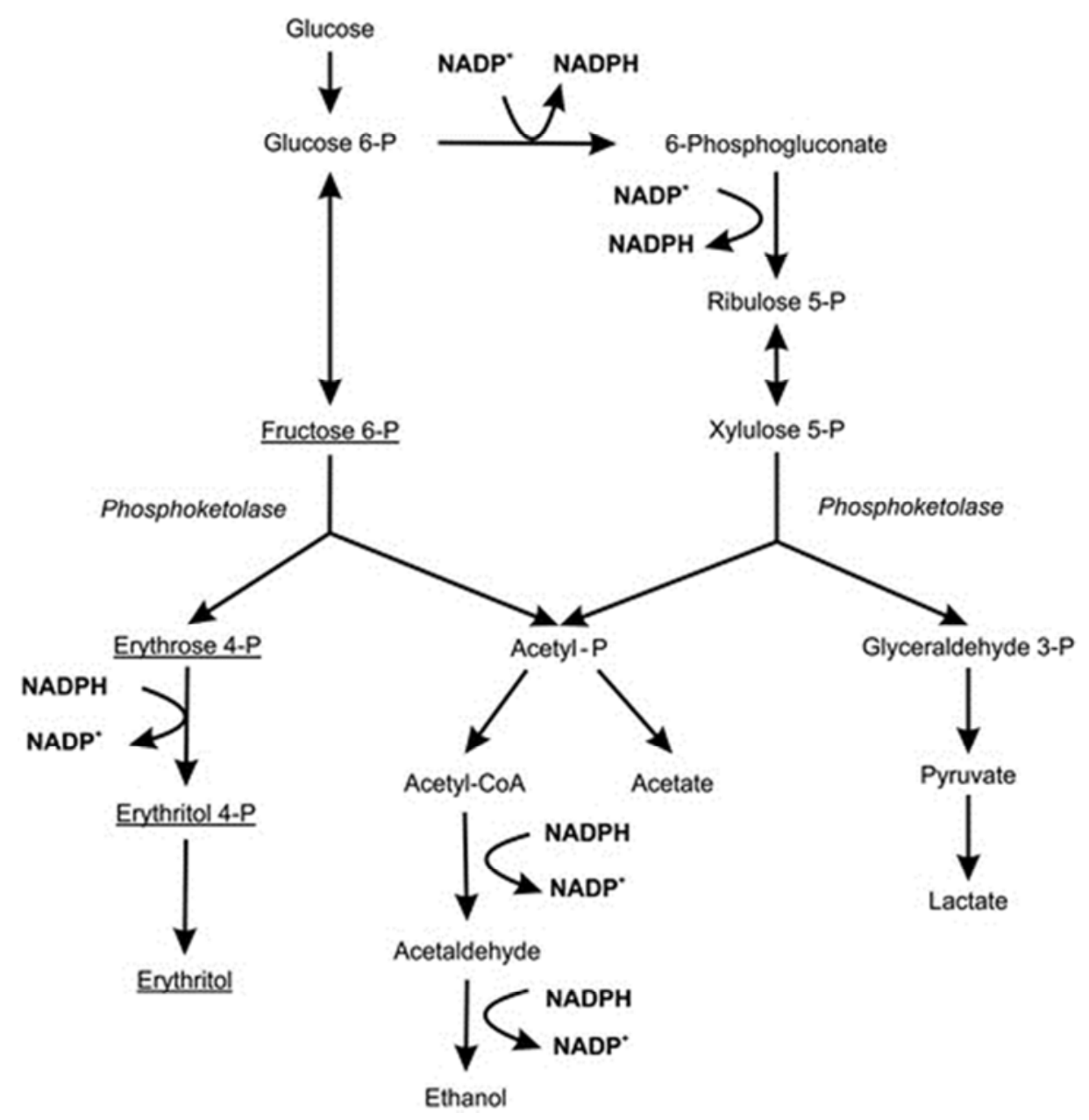

Figure 8. Phosphoketolase pathway.

High NADPH / NADP ratio in the pathway inhibits the enzyme glucose 6-phosphate dehydrogenase. This inhibition causes the accumulation of glucose-6-P for the isomerization into fructose-6-P which is cleaved into erythrose-4-P, and Acetyl-2-P, Erythrose 4-P is dephosphorylated and reduced into erythritol [31].

\subsection{Metabolic Engineering}

Erythritol biosynthesis is multiple steps pathway with multiple enzymes and enzymatic reactions generating intermediate metabolites in the process. These intermediate metabolites in erythritol biosynthesis are undesirable byproducts, effect erythritol yield and productivity. In addition, these intermediate metabolites at the end of fermentation process interfere in erythritol recovery process causing low erythritol recovery efficiency.

The strategy to Inhibit the formation and accumulation of these intermediate metabolites in erythritol biosynthesis is very important factor to improve erythritol recovery efficiency and yield at lower cost. One of these intermediate metabolites is the organic acid fumarate that inhibit the enzyme erythrose reductase which is the key enzyme for the reduction of generated five carbon aldehyde sugar erythrose into the five-carbon sugar alcohol erythritol [24]. The inhibition of fumarate biosynthesis in fermentation process is being achieved by the addition of $\mathrm{Cu}^{+}$in the form of copper sulphate in fermentation medium [25]. Other intermediate metabolites biosynthesis can be reduced or eliminated by specific enzymatic inhibition or by metabolic pathway engineering technology.

Osmotic stress factor in erythritol fermentation process condition is important factor for high erythritol yield with lower intermediate metabolites from osmophilic yeasts, or osmophilic yeasts-like fungi erythritol producer. This osmotic stress factor in fermentation process is transmitted through the high osmolarity glycerol (HOG) pathway [26]. The definition of HOG pathway is the adaptation of yeast or other eukaryotic cells to hyperosmotic stress regulated by mitogen-activated protein kinase (MARK). Protein kinase is specific enzyme for the phosphorylation of the two amino acids serine and threonine [27]. Phosphorylation of these two amino acids serine and threonine trigger signal transduction to regulate cells functions, metabolism, and gene expression [28], One of HOG pathway is cells function for osmotic stress regulation in erythritol biosynthesis pathway at higher yield from osmophilic yeasts or yeasts-like fungi in fermentation process. This function of osmotic stress factor occurred due to the accumulation of intracellular small molecules with a neutral charge known by the name osmolytes. These osmolytes are responsible to balance the osmotic pressure in both inner and outer microbial cell membrane and prevent the efflux of water 
[29]. In the case of osmophilic yeasts and yeasts-like fungi producing erythritol, the small molecule (osmolyte) is glycerol that accumulate inside yeast cells. It is demonstrated that the Inhibition of HOG pathway by the deletion of the DNA gene caries osmo-protection in yeast cells resulted in a mutant of osmo-sensitive yeast that cannot survive at high carbon source (e.g, glucose, or glycerol) or produce erythritol in fermentation process [30]. This developed mutant of osmo-sensitive yeast demonstrated the important of osmotic stress factor for the production of high erythritol yield at high conversion rate from high concentration of carbon source in fermentation medium. It is important to highlight, that all industrial yeasts or yeasts-like fungi cultures producing erythritol such as Moniliella megachiliensis, Candida magnoliae, Yarrowia lipolytica, and Torula sp. are osmophilic cultures due to this high osmolarity glycerol (HOG) pathway.

Fermentation process for the production of higher erythritol yield with less intermediate metabolites from these osmophilic yeasts or yeasts-like fungi can be achieved by metabolic pathway engineering to develop strains overexpress high activities of key enzymes in pentose-phosphate pathway for higher conversion of glucose or other carbo source into erthrose-4-P, and also, overexpress high activity of the two enzymes phosphatase and erythrose reductase that are not key enzymes in pentose phosphate pathway and are responsible for dephosphorylation of erythrose-4-P into erythrose, and the conversion of erythrose into erythritol [31].

It is observed in fermentation process that the depletion of the carbon source (e.g., glucose, fructose, or glycerol) in erythritol production resulted in the utilization of produced erythritol as a carbon source by these industrial osmophilic microbial cultures. The utilization of erythritol as a carbon source by these industrial osmophilic microbial cultures is due to erythritol catabolic pathway [32]. Enzymes responsible for erythritol catabolic pathway are erythritol dehydrogenase, kinases, and isomerases [33]. The deletion of the DNA (genes) for these catabolic pathway key enzymes in industrial osmophilic microbial cultures resulted in the inhibition of erythritol utilization by catabolic pathway and improve erythritol yield and productivity [34].

This metabolic engineering technology is currently used for the modification of biosynthesis pathways in industrial microorganisms to improve the yield and productivity of targeted products in fermentation process. Metabolic engineering is microbial manipulation methods by mutation or by genetic engineering. Microbial mutation can be induced by physical methods such as ultraviolet (UV) or by chemicals methods such as nitrosoguanidine (NTG), followed by microbial screening for the selection of mutant or mutants producing high yield and productivity of a target product in shorter time [35]. Genetic engineering is faster approach and is used to enhance target product productivity, reduce undesired intermediate metabolites, and to utilize wide range of raw materials as carbon sources in biosynthesis pathway [36].

These metabolic engineering methods by microbial mutation or by genetic engineering are currently applied on industrial osmophilic yeasts or yeasts-like fungi for pentose phosphate pathway modification to improve erythritol biosynthesis in fermentation process from cheap carbon sources with less intermediate metabolites such as mannitol, fumarate, glycerol, acetyl-P, ribitol, and arabitol [37]. These intermediate metabolites interfere in erythritol productivity and recovery.

Other metabolic engineering application is the expression of new specific enzymes in erythritol producing microorganisms to utilize wide range of polysaccharides as a carbon source in fermentation process. As an example, the expression of cellulase and hemicellulase enzymes in industrial microorganisms to utilize a cheap lignocellulosic materials as a carbon source in fermentation process in replacement to expensive sugars such as glucose to lower the production cost of erythritol. [38].

\section{Erythritol Production}

Commercial erythritol production on large scale is by biotechnology processes that include; medium preparation, inoculum preparation, fermentation process, and erythritol recovery process (figure 9).

\section{Fermentation medium:}

The composition of fermentation medium for erythritol production is vary based on the industrial microbial type (genus, species, and strain) used in fermentation process. In general, the common fermentation medium for erythritol production by osmophilic yeasts or yeasts-like fungi is carbon source mainly glucose ( $>200 \mathrm{gm} / \mathrm{L})$, nitrogen source mainly yeasts extract $(\geq 12 \mathrm{mg} / \mathrm{L})$ or ammonium sulphate $(\geq 2.5 \mathrm{gm}$ $/ \mathrm{L})$, sodium chloride ( $\geq 20 \mathrm{gm} / \mathrm{L})$, monopotassium phosphate $(\geq 0.25 \mathrm{gm} / \mathrm{L})$, this in addition to other additives of minerals, vitamins, and surfactant [39]. Minerals in fermentation medium are copper, manganese, and zinc in the form of copper sulfate $\mathrm{CuSO}_{4} \cdot 5 \mathrm{H}_{2} \mathrm{O}(\geq 2 \mathrm{mg} / \mathrm{L})$, manganese sulfate $\mathrm{MnSO}_{4} \cdot 4 \mathrm{H}_{2} \mathrm{O}(\geq 10 \mathrm{mg} / \mathrm{L})$, and zinc sulphate $\mathrm{ZnSO}_{4},(\geq$ $0.25 \mathrm{mM})$. These minerals at selected optimum concentrations increase erythritol production yield [40]. Vitamins such as thiamin and inositol in fermentation medium are important for microbial growth and erythritol biosynthesis [41]. The vitamin thiamin is present in yeast extract added in fermentation medium as a nitrogen source. Some manufacturer replaces the costly yeast extract in fermentation medium with a cheap nitrogen source such as ammonium sulphate $\left(\mathrm{NH}_{4}\right)_{2} \mathrm{SO}_{4}$. In the case of using ammonium salt as a nitrogen source in fermentation medium, adding thiamin is nesseacary. The vitamin inositol in fermentation medium is also important factor for erythritol biosynthesis. Surfactants such as Triton $\mathrm{X}-100$, Tween80, or Span20 is commonly used in fermentation medium as antifoam added during fermentation process as needed to inhibit foam formation due to fermentation process of aeriation, and agitation. High foam in fermenter effect oxygen transfer and erythritol yield [42].

Glucose as carbon source at high contention ( $\geq 200 \mathrm{gm} / \mathrm{L})$ plus salts such as sodium chloride $(\geq 20 \mathrm{gm} / \mathrm{L})$ is added in fermentation medium to provide the high osmotic stress factor 
in fermentation process for erythritol yield and productivity by industrial osmophilic yeasts or yeasts-like fungi [43]. Other important factor in fermentation medium, is the optimum carbon to nitrogen ratio $(\mathrm{C}: \mathrm{N})$. This ratio is usually at the rate of 80:1. Finally, the optimum fermentation process conditions of temperature, $\mathrm{PH}$, aeriation, and agitation are vital factors for higher bioconversion rate of carbon source into erythritol with minimum intermediate metabolites biosynthesis and accumulation as byproducts [44].

\section{Inoculum preparation}

Industrial microorganisms that are used for commercial erythritol manufacturing are osmophilic yeasts or yeasts-like fungi of Moniliella pollinis, Trichosporonoides megachiliensis, and recently Yarrowia lipolytica. Inoculum preparation (figure-9) from these industrial cultures is started from lyophilized cells to inoculate laboratory shaker flask, the growth in shaker flask is transferred into inoculum vessel (I-vessel), the growth in the I-vessel is transferred into seed vessel (S-vessel). Finally, the growth in S-vessel is transferred into production scale fermenter (F-vessel). The aim from these multiple propagation stages for inoculum preparation is to produce high inoculum cells mass $(\sim 10 \%$ volume $)$ in the production fermenter (F-vessel) to reduce the lag phase in cells growth curve for shorter fermentation time and higher yield of erythritol production [45].

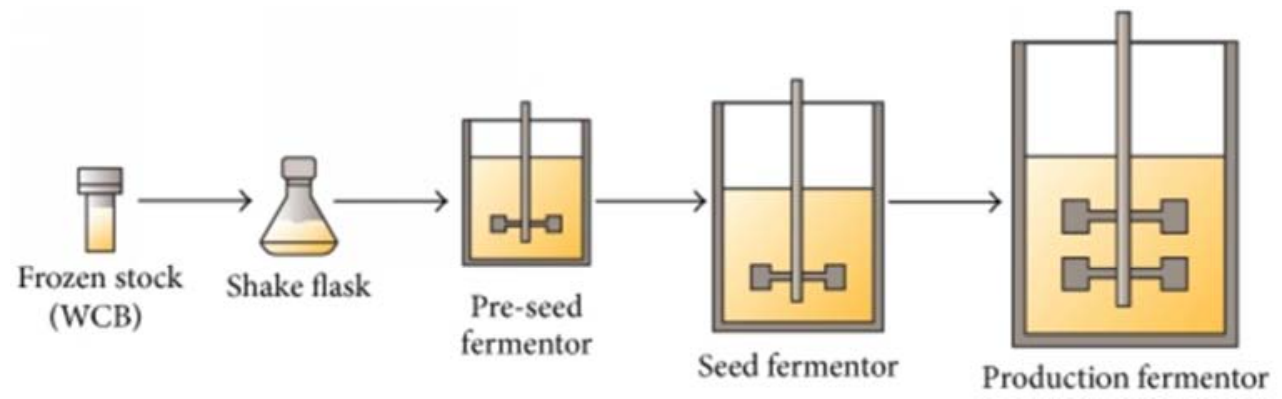

Figure 9. Diagram for fermentation process steps.

Steps started from frozen culture used to inoculate shake flask, the growth in the shake flask is used to inoculate pre seed fermenter (I-vessel), the growth in pre seed fermenter is used to inoculate the production fermenter (F-vessel) [52].

\section{Fermentation process methods}

There are different fermentation process methods that are commonly used on large scale operations. These are batch fermentation, fed-batch fermentation, and continuous fermentation.

Batch fermentation method, is closed fermentation system to the end, with no addition added, and ended upon the depletion of nutrients. This batch fermentation process is the simplest method for operation, and does not require higher operation attention as in the case for other fermentation process methods.

Fed-batch fermentation method is a system were extra amount of the carbon source such as glucose is added for at least once during fermentation process. This fed-batch fermentation method improves the target yield and productivity comparing to batch fermentation method and it is the process method that is commonly used for erythritol production on large scale [46].

Continuous fermentation method started as a batch fermentation method, and as soon cell growth is reached the exponential phase, continuous feeding the fermenter with fresh medium is started and in the same time continuous removing of equal amount of fermentation broth for recovery process. This continuous fermentation process method showed no success for erythritol production.

Recovery process

Erythritol recovery process from fermentation broth is multiple steps [47] of filtration to separate the microorganism from the fermentation broth, ion exchange chromatography to separate erythritol in the fermentation broth from byproducts, decolorization of separated erythritol fraction using activated charcoal, and finally the decolorized erythritol fraction is concentrated and crystallized for packaging, and marketing.

Erythritol product is marketed in the form of powder or granules with the following general specification:

$\begin{array}{ll}\text { Items } & \text { Specification } \\ \text { Appearance } & \text { White crystalline powder } \\ \text { Melting Point } & 118 \sim 122 \\ \mathrm{pH} & 5.0 \sim 7.0 \\ \text { Moisture } & \leq 0.20 \% \\ \text { Ash } & \leq 0.01 \% \\ \text { As } & \leq 2.0 \mathrm{mg} / \mathrm{kg} \\ \text { Heavy Metals } & \leq 5.0 \mathrm{mg} / \mathrm{kg} \\ \text { Assay (on dry basis) } & \geq 99.0 \% \\ \text { Total Plate Count } & \leq 1000 \mathrm{cfu} / \mathrm{g} \\ \text { E. coli } & \text { Negative } \\ \text { Salmonella } & \text { Negative }\end{array}$

\section{Global Erythritol Market Size}

Current erythritol global market size is exceeded \$ 195 million [48], and the global market demand continue to increase at the rate of $6.5 \%$ CAGR. It is expected global erythritol market to reach about $\$ 310$ million by the year 2026 . This large market size demand is due to increase awareness toward the use of healthy diets as low-calorie sugar substitutes, plus erythritol applications in food, pharmaceuticals, and bio-chemical industries. 


\section{Discussion}

The biosynthesis of erythritol by osmophilic yeasts or yeasts-like fungi is multiple steps of enzymatic reactions in pentose- phosphate partway. This pathway is consisting of two phases.

The first phase is oxidative pathway with the end product of ribulose-5-phoshate. This phase is irreversible and depends on the reduction of $\mathrm{NADP}^{+}$into NADPH to generate the energy needed for enzymatic reaction to convert glucose-6-P into ribulose -5-P. The second phase is the non-oxidative pathway started from ribulose-5-P to covet into erytrose-4-P in multiple steps of reversable enzymatic reactions.

Key enzymatic activities for these two phases are glucose-6-phosphate dehydrogenase, phosphogluconate dehydrogenase, transketolase, and transaldolase. These key enzymes are responsible for the conversion of glucose into erythrose-4-P in fermentation process by osmophilic yeasts or yeasts-like fungi cultures such as Moniliella megachiliensis., Candida magnolia, or Yarrowia lipolytica. These industrial microbial cultures are capable to dephosphorylate erythrose-4-P into erythrose and express the enzyme erythrose reductase to converted dephosphorylated aldehyde four carbon sugar erythrose into sugar alcohol erythritol in the presence of the co-enzyme NADH.

In addition, to these industrial microbial cultures of osmophilic yeasts and yeasts-like fungi, some filamentous fungi such as Trichoderma reesei demonstrated the ability to produce low erythritol yield from cheap lignocellulosic materials. These filamentous fungi secrete cellulase and hemicellulase enzymes that breakdown lignocellulosic materials into monosaccharides, and convert these monosaccharides into low yield of erythritol via pentose phosphate pathway. The secretion of lignocellulosic enzymes by these filamentous fungi is an advantage for potential production higher yield of erythritol by fermentation process from cheap lignocellulosic materials as a carbon source in replacement to the expensive glucose. Improve erythritol biosynthesis in these filamentous fungi can be achieved by metabolic engineering through microbial mutation or genetic engineering. This metabolic engineering technology is the key for developing industrial strains from these filamentous fungi expressing higher enzymatic activities to hydrolyze lignocellulosic materials into monosaccharides and to convert monosaccharides into erythritol at higher conversion rate in shorter time and lower cost.

In addition, to these filamentous fungi, some heterofermentative lactic acid bacteria such as Leuconostoc oenos showed the capability to produce low erythritol yield from glucose via phosphoketolase pathway. These heterofermentative lactis acid bacteria under anaerobic or aerobic fermentation process convert glucose into lactic acid, acetates, ethanol, and low concentrations of sugar alcohols including erythritol. Metabolic pathway engineering is also the technology to improve key enzymatic activities in phosphoketolase pathway for higher rate of erythritol biosynthesis, and to inhibit or deactivate key enzymes responsible for the biosynthesis and accumulation of lactic acid, acetates, and ethanol as intermediate metabolites. Inhibition the of these intermediate metabolites in erythritol biosynthesis is the industrial approach to direct the conversion of glucose toward the biosynthesis of erythritol in the fermentation process at higher yield from these heterofermentative lactic acid bacteria.

It is also, demonstrated that the expression of high ratio of co-enzymes NADH/NADP ${ }^{+}$in phosphoketolase pathway for heterofermentative lactic acid bacteria inhibit the enzymatic activity of glucose-6-dehydrogenase resulted in the accumulation of glucose-6-P for isomerization into fructose-6-P at higher rate. This fructose-6-P is the substrate for the conversion into erythrose-4-P, and acetyl-2-P by the enzyme phosphoketolase. The accumulated erythrose-4-P in heterofermentative lactic acid bacteria cells is dephosphorylated by phosphatase enzyme into erythrose and reduced into erythritol by erythrose reductase enzyme at higher erythritol yield with less intermediate metabolites of lactate, acetate, and ethanol.

In Summary, metabolic engineering technology for developing industrial microbial culture producing high erythritol yield from carbon sources requires the identification and sequencing key enzymes DNA (genes) in erythritol biosynthesis pathway, overexpressing identified key enzymes DNA (genes) in the target microorganism to enhance enzymatic activities in metabolic pathways toward higher conversion rate of carbon sources into erythritol. This metabolic engineering technology also used to inhibit or delete key enzymes DNA (genes) for the biosynthesis of undesirable intermediate metabolites that interfere in erythritol biosynthesis. In general, this metabolic engineering technology is an attractive strategy and is currently applied to improve erythritol production by fermentation process from osmophilic yeasts and yeasts-like fungi. This metabolic engineering technology is, also, the strategy for future large scale erythritol production from filamentous fungi, and from heterofermentative lactic acid bacteria by fermentation process.

Fermentation process for commercial production of erythritol from osmophilic yeasts and yeasts-like fungi, required the operation under high osmotic pressure. This high osmotic pressure is the stress factor that influence metabolic pathway in yeast cells growth toward erythritol biosynthesis with less intermediate metabolites. This osmotic pressure as stress factor in fermentation process can be achieved by high molar concentration of the carbon source (sugar) in fermentation medium.

Carbon sources as a substrate, and produced erythritol have different molar masses (gram / liter) in fermentation medium. These molar masses are important factor for calculating the optimum osmotic pressure in fermentation process. Other factor is chemical interaction of fermentation medium components specially carbon and nitrogen sources during sterilization. This chemical interaction during fermentation medium sterilization might change the initial carbon source concentration and effect the osmotic pressure in fermentation 
process. Kinetic analysis for erythritol production by fermentation is very important factor to achieve the optimum osmotic pressure in fermentation process. This kinetic analysis can be performed by measuring the osmolarity value of $0.5 \mathrm{M}$ carbon source (sugars) and the osmolarity value of $0.5 \mathrm{M}$ of end product erythritol. From these measurements' initial concentration of carbon source in fermentation medium can be calculated. These measurements are also used to calculate and readjust osmotic pressure during fermentation process by the addition of the carbon source into fermentation medium as needed.

Fed-batch fermentation method for erythritol production demonstrated to be the best method to control osmotic pressure during fermentation process. In this method, the operator is able to calculate carbon source concentration (molar mass) in fermentation process, and feed the fermenter with the carbon source as needed to maintain the optimum osmotic pressure required for higher erythritol yield and productivity.

Minerals, and vitamins at optimum concentrations in fermentation medium also plays an important role for high erythritol yield and productivity. Minerals such as copper $\left(\mathrm{Cu}^{2+}\right)$, manganese $\left(\mathrm{Mn}^{2+}\right)$, and zinc $\left(\mathrm{Zn}^{2}\right)$ are enzymes co-factors for erythritol biosynthesis. $\mathrm{Mn}^{2}$, is important factor for the activity of the enzyme erythrose reductase, $\mathrm{Zn}^{2}$ increase microbial cells permeability for the secretion of intracellular erythritol outside cells to the fermentation broth. $\mathrm{Cu}^{2+}$ inhibits biosynthesis for mannitol, fumarate, and other intermediate metabolites in citric acid pathway, these intermediate metabolites interfere in erythritol biosynthesis and recovery. Minerals at the optimum concentrations in fermentation medium improve bioconversion efficiency of carbon sources into erythritol. Vitamins such as thiamin and inositol at optimum concentrations in fermentation medium contribute to microbial cell growth and erythritol biosynthesis rate. Inositol is also, enhance the activity of the enzyme erythrose reductase to convert erythrose into erythritol in the presence of NADH. It is important to highlight, that optimum concretions of these minerals and vitamins in fermentation medium are varied depend on microbial culture type (genus, species, and strain) used in fermentation process for erythritol production.

Finally, improve erythritol production yield by fermentation process depends on multiple factors. These factors are but not limited to the selection of microbial culture, improve selected microbial culture for higher erythritol productivity by microbial mutation, or genetic engineering, design fermentation medium with optimum concentrations of nutrients for higher microbial growth rate and erythritol productivity, selection the optimum fermentation conditions of aeration, and agitation for higher growth rate, monitor fermentation process for $\mathrm{PH}$, and temperature to the optimum conditions to improve industrial culture growth rate and erythritol productivity, monitor the conversion of carbon source into erythritol by chemical analysis, control the optimum osmotic pressure by feeding the fermenter with carbon source (sugars) as needed, and the selection of optimum recovery process for higher erythritol recovery efficiency. All these are important production factors to achieve higher conversion rate of carbon sources into higher erythritol yield at lower cost.

\section{Conclusion}

Erythritol manufacturing is complicated process comparing to other sugar alcohols manufacturing. This complicated process is due to multiple enzymes and enzymatic reactions in metabolic pathways for erythritol biosynthesis from carbon sources. In addition, these metabolic pathways generate intermediate metabolites that interfere in erythritol biosynthesis rate and recovery. These intermediate metabolites in the erythritol pathways can be reduced or eliminated by metabolic engineering technology.

\section{References}

[1] K. Regnat, R. L. Mach, and A. R. Mach-Aigner: Erythritol as sweetener-wherefrom and where to?, Appl Microbiol Biotechnolology. 102 (2): 587-595 (2018).

[2] Sheng-Dunlin, Ching-Ching Lee, Jeng-Leun Mau, Li-Yun Lin, and Shiow-Ying Chiou: Effect of erythritol on quality characteristic of reduced -calorie Danish cookies Journal of Food Quality 33 14-26 (2010).

[3] Peter Decock, and Claire-Lise Bechert: Erythritol. Functionality in noncaloric functional beverages Pure and Applied Chemistry 74 (7): 1281-1289 (2002).

[4] M Hiele, Y Ghoos, P Rutgeerts, and G Vantrappen: Metabolism of erythritol in humans: comparison with glucose and lactitol British Journal of Nutrition 69 (1): 169-76 (1993).

[5] GRAS notices: erythritol. US Food and Drug Administration. 16 November 2018. Retrieved 8 December 2018.

[6] Shinji Ohmori, Yasuo Ohno, Tadashi Makino, and Toshio Kashihara: Characteristics of erythritol and formulation of a novel coating with erythritol termed thin-layer sugarless coating, International Journal of Pharmaceutics 278 (2), 8 447-457 (2004).

[7] Daniëlle M. P. H. J. Boesten, Gertjan J. M. den Hartog, Peter de Cock, Douwina Bosscher, Angela Bonnema, and Aalt Bast: Health effects of erythritol Nutrafoods 14: 3-9 (2015).

[8] Sloan JW, Hofreiter BT, Mehltretter CL, and Wolff IA: Hydrogenolysis of dialdehyde starch to erythritol and ethylene glycol. US Patent US 2,783, 283 (1957).

[9] Shie-Jea Lin, Chiou-Yen Wen, Jian-Ching Liau, and Wen-Shen $\mathrm{Chu}$ : Screening and production of erythritol by $\mathrm{j}$ isolated osmophilic yeast-like fungi, Process Biochemistry 36 (12): 1249-1258 (2001).

[10] Lloyd NE, Nelson WJ. In: Whistler RL, BeMiller JN, Paschall EF, eds. Starch Chemistry and Technology. Orlando, FL: Academic Press; 611-660 (1984).

[11] Laura-Katharina Bertels, Lucía Fernández Murillo, and Jürgen J. Heinisch: The Pentose Phosphate Pathway in Yeasts-More Than a Poor Cousin of Glycolysis, Biomolecules. 11 (5): 725 (2021). 
[12] Christopher C. Steel*, Paul R. Grbin', Alan W. Nichol: The pentose phosphate pathway in the yeasts Saccharomyes cerevisiae and Kloeckera apiculata, an exercise in comparative metabolism for food and wine science students. Biochemistry and Molecular Biology Education 29: 245-249 (2001).

[13] Nicholas JKruger, and Antjevon Schaewen: The oxidative pentose phosphate pathway: structure and organization, Current Opinion in Plant Biology 6 (3): 236-246 (2003).

[14] Huiling Cheng, Siqi Wang, Muhammad Bilal, Xuemei Ge, Can Zhang, Patrick Fickers, and Hairong Cheng: Identification, characterization of two NADPH-dependent erythrose reductases in the yeast Yarrowia lipolytica and improvement of erythritol productivity using metabolic engineering, Microbial Cell Factories. 17: 133 (2018).

[15] Ji-Hee Yu, Dae-Hee Lee, Yong-Joo Oh, Ki-Cheol Han, Yeon-Woo Ryu, and Jin-Ho Seo: Selective utilization of fructose to glucose by Candida magnoliae, an erythritol producer, Applied Biochemistry and Biotechnology 131: 870-879 (2006).

[16] Marina A. Y. Aoki, Glaucia M. Pastore, and Yong K. Park: Microbial transformation of sucrose and glucose to erythritol, Biotechnology Letters 15: 383-388 (1993).

[17] Ludwika Tomaszewska, Anita Rywińska, and Witold Gładkowski: Production of erythritol and mannitol by Yarrowia lipolytica yeast in media containing glycerol. Journal of Industrial Microbiology \& Biotechnology 39: 1333-1343 (2012).

[18] Ludwika Tomaszewska, Magdalena Rakicka, Waldemar Rymowicz, and Anita Rywińska: A comparative study on glycerol metabolism to erythritol and citric acid in Yarrowia lipolytica yeast cells, FEMS Yeast Research, 14 (6): 966-976 (2014).

[19] Alexandra Mirończuk, Anna Biegalska, and Adam Dobrowolski.: Functional overexpression of genes involved in erythritol synthesis in the yeast Yarrowia lipolytica. Biotechnol Biofuels. 10: 77 (2017).

[20] Birgit Jovanović, Robert L Mach, and Astrid R Mach-Aigner: Erythritol production on wheat straw using Trichoderma reesei AMB Express 4: 34 (2014).

[21] Huiling Cheng, Siqi Wang, Muhammad Bilal, Xuemei Ge, Can Zhang, Patrick Fickers, and Hairong Cheng: Identification, characterization of two NADPH-dependent erythrose reductases in the yeast Yarrowia lipolytica and improvement of erythritol, Microbial Cell Factories 17: 133 (2018).

[22] C A Tyler, L Kopit, C Doyle, A O Yu, J Hugenholtz, and M L Marco: Polyol production during heterofermentative growth of the plant isolate Lactobacillus florum2F. J Appl Microbiol. 120: 1336-1345 (2016)

[23] M. Veiga-da-Cunha, H. Santos, and E. Van Schaftingen: Pathway and regulation of erythritol formation in Leuconostoc oenos, J. Bacteriology 175 (13) 3941-3941 (1993).

[24] Jung-Kul Lee, Bong-Seong Koo, and Sang-Yong Kim: Fumarate-Mediated Inhibition of Erythrose Reductase, a Key Enzyme for Erythritol Production by Torula corallina, Appl Environ Microbiol. 68 (9): 4534-4538 (2002).

[25] Jung-Kul Lee, Suk-Jin Ha, Sang-Yong Kim, and Deok-Kun Oh: Increased erythritol production in Torula sp. by $\mathrm{Mn}^{+}$and $\mathrm{Cu} 2^{+}$, Biotechnology Letters 22 (12): 983-986 (2000).
[26] Li-BoYangXiao-BeiZhan, Zhi-YongZhengJian-Rong Wu, and Min-JieGaoChi-ChungLin: A novel osmotic pressure control fed-batch fermentation strategy for improvement of erythritol production by Yarrowia lipolytica from glycerol, Bioresource Technology 151: 120-127 (2014).

[27] Liang Shi, Malcolm Potts, Peter J. Kennelly: The serine, threonine, and/or tyrosine-specific protein kinases and protein phosphatases of prokaryotic organisms: a family portrait FEMS Microbiology Reviews, 22: (4) 229-253 (1998).

[28] Dorota A. Rzechonek, Alison M. Day, Janet Quinn, and Aleksandra M. Mirończuk: Influence of ylHog1 MAPK kinase on Yarrowia lipolytica stress response and erythritol production, Scientific Reports (8) 14735 (2018).

[29] Stefan Hohmann: Osmotic Stress Signaling and Osmoadaptation in Yeasts, Microbiology and Molecular Biology Reviews. 66 (2): 300-372 (2002).

[30] Dorota A. Rzechonek, Mateusz Szczepańczyk, Guokun Wang, Irina Borodina, and Aleksandra M. Mirończuk: HOG-Independent Osmoprotection by Erythritol in Yeast Yarrowia lipolytica, Genes (Basel). 11 (12): 1424 (2020).

[31] Dorota A. Rzechonek, Adam Dobrowolski, Waldemar Rymowicz and Aleksandra M. Mironczuk: Recent advances in biological production of erythritol, Critical reviews in Biotechnology 38 (4) 620-633 (2018).

[32] Frédéric Carlya, Marie Vandermiesb, Samuel Telekb, Sébastien Steelsb, Stéphane Thomasc, Jean-Marc Nicaudc, Patrick Fickersb: Enhancing erythritol productivity in Yarrowia lipolytica using metabolic engineering, Metabolic Engineering, 42: 19-24 (2017).

[33] J. F. Sperry, D C Robertson: Erythritol catabolism by Brucella abortus 1975121 (2): 619-30 (1975).

[34] Katsuhiko Sawada, Arihiro Taki, Takashi Yamakawa, and Minoru Seki: Key role for transketolase activity in erythritol production by Trichosporonoides megachiliensis SN-G42 108 (5): 385-90 (2009).

[35] Justin P. Hawkins, Patricia A. Ordonez and Ivan J. Oresnik: Characterization of Mutations That Affect the Nonoxidative Pentose Phosphate Pathway in Sinorhizobium meliloti, 200 (2): e00436-17.

[36] Aniek Dvander Woude, Ruth Perez Gallego, Angie Vreugdenhil, Vinod Puthan Veetil, Tania Chroumpi, and Klaas J. Hellingwerf: Genetic engineering of SynechocystisPCC6803 for the photoautotrophic production of the sweetener erythritol, Micrboial Cell Fact 15: 60 (2016).

[37] Laxman S. Savergave, Ramchandra V. Gadre, Bhalchandra K. Vaidya, and Karthik Narayanan: Strain improvement and statistical media optimization for enhanced erythritol production with minimal by-products from Candida magnoliae mutant R23, Biochemical Engineering Journal, 55 (2): 92-100 (2011).

[38] Magdalena Rakicka, Anna Biegalska, Waldemar Rymowicz, Adam Dobrowolski, and Aleksandra M. Mirończuk: Polyol production from waste materials by genetically modified Yarrowia lipolytica. Bioresource Technology, 243: 393-399 (2017).

[39] G. J. Hajny, J. H. Smith, and J. C. Garver: Erythritol Production by a Yeastlike Fungus, Applied Microbiology. 12 (3): 240-246 (1964). 
[40] Ludwika Tomaszewska, Waldemar Rymowicz, and Anita Rywińska: Mineral Supplementation Increases Erythrose Reductase Activity in Erythritol Biosynthesis from Glycerol by Yarrowia lipolytica, Applied Biochemistry and Biotechnology 172): 3069-3078 (2014).

[41] Jung-Kul Lee, Suk-Jin Ha, Sang-Yong Kim, and Deok-Kun Oh: Increased erythritol production in Torula sp. with inositol and phytic acid, Biotechnology Letters 23: 497-500 (2001).

[42] Magdalena Rakicka, Anita Rywińska Krzysztof Cybulski, and Waldemar Rymowicz: Enhanced production of erythritol and mannitol by Yarrowia lipolytica in media containing surfactants, Brazilian Journal of Microbiology 47: (2) 417-423 2016.

[43] Shie-Jea Lin, Chiou-Yen Wen, Pei-ming Wang, and Wen-Shen Chu: High-level production of erythritol by mutants of osmophilic Moniliella sp, Process biochemistry 45 (6): 973-979 (2010).

[44] Y- W Ryu, CY Park, JB Park, S-Y Kim and J- H Seo: Optimization of erythritol production by Candida magnoliae in fed-batch culture, Journal of Industrial Microbiology \& Biotechnology 25, 100-103 (2000).

[45] Saurabh Saran, Sanjana Mukherjee, Jyotsana Dalal, and Rajendra Kumar Saxena: High production of erythritol from Candida sorbosivorans SSE-24 and its inhibitory effect on biofilm formation of Streptococcus mutans. Bioresource Technol 198: 31-38 (2015).

[46] Aleksandra M Mirończuk, Joanna Furgała, Magdalena Rakicka, Waldemar Rymowicz: Enhanced production of erythritol by Yarrowia lipolytica on glycerol in repeated batch cultures Journal of Industrial Microbiology and Biotechnology 41 (1) 57-64 (2014).
[47] Siqi Wang, Hengwei Wang, Jiyang Lv, Zixin Deng, and Hairong Cheng: Highly Efficient Erythritol Recovery from Waste Erythritol Mother Liquor by a Yeast-Mediated Biorefinery Process, Journal and Agriculture Food Chemistry. 65 (50), 11020-11028 (2017).

[48] Erythritol Sweetener Market Size 2021: Growth Analysis by Development Trends, Business Demand Status and Global Share Forecast to 2026. Research report, March 7th (2021).

[49] Yvette G. T. Loeffen, Nathalie Biebuyck, Mirjam M. C. Wamelink, Cornelis Jakobs, Margot F. Mulder, Anna Tylki-Szyman' ska, Cheuk-Wing Fung, Vassili Valayannopoulos, and Arend Bo kenkamp: Nephrological abnormalities in patients with transaldolase deficiency, Nephrology Dialysis Transplantation 0: 1-5 (2012).

[50] Widayat, Agam Duma Kalista Wibowo, and Hadiyantoa: Study on production process of biodiesel from rubber seed (hevea brasiliensis) by in situ (trans) esterification method with acid catalyst, Energy Procedia 32: 64 - 73 (2013).

[51] Frédéric Carly, and Patrick Fickers: Erythritol production by yeasts: a snapshot of current knowledge. Yeast 35: (7) 455-463 (2018).

[52] Victor Fedorenko, Olga Genilloud, Liliya Horbal, Giorgia Letizia Marcone,, Flavia Marinelli, Yossi Paitan, and Eliora Z. Ron, Antibacterial Discovery and Development: From Gene to Product and Back. Journal of Biomedicine and Biotechnology $\cdot$ September 2015. 